\title{
Different Forms of Self-Control Share a Neurocognitive Substrate
}

\author{
Golnaz Tabibnia, ${ }^{1}$ John R. Monterosso, ${ }^{1}$ Kate Baicy, ${ }^{1}$ Adam R. Aron, ${ }^{2}$ Russell A. Poldrack, ${ }^{2,3}$ Shruthi Chakrapani, ${ }^{1}$ \\ Buyean Lee, ${ }^{1}$ and Edythe D. London ${ }^{1,3,4}$ \\ Departments of ${ }^{1}$ Psychiatry and Biobehavioral Sciences, ${ }^{2}$ Psychology, and ${ }^{3}$ Molecular and Medical Pharmacology, and ${ }^{4}$ Brain Research Institute, University \\ of California, Los Angeles, California 90024
}

Psychological and neurocognitive studies have suggested that different kinds of self-control may share a common psychobiological component. If this is true, performance in affective and nonaffective inhibitory control tasks in the same individuals should be correlated and should rely upon integrity of this region. To test this hypothesis, we acquired high-resolution magnetic resonance images from 44 healthy and 43 methamphetamine-dependent subjects. Individuals with methamphetamine dependence were tested because of prior findings that they suffer inhibitory control deficits. Gray matter structure of the inferior frontal gyrus was assessed using voxel-based morphometry. Subjects participated in tests of motor and affective inhibitory control (stop-signal task and emotion reappraisal task, respectively); and methamphetamine-dependent subjects provided self-reports of their craving for methamphetamine. Performance levels on the two inhibitory control tasks were correlated with one another and with gray matter intensity in the right pars opercularis region of the inferior frontal gyrus in healthy subjects. Gray matter intensity of this region was also correlated with methamphetamine craving. Compared with healthy subjects, methamphetamine-dependent subjects exhibited lower gray matter intensity in this region, worse motor inhibitory control, and less success in affect regulation. These findings suggest that self-control in different psychological domains involves a common substrate in the right pars opercularis, and that successful self-control depends on integrity of this substrate.

\section{Introduction}

Previous studies have suggested that different kinds of self-control may share a common psychobiological component (Muraven and Baumeister, 2000; Cohen and Lieberman, 2010). Self-control in these studies refers to the process of inhibiting an otherwise imminent action, thought, or feeling (i.e., motor, cognitive, or affective inhibitory control). If self-control in different psychological domains indeed draws from a common psychobiological resource, then (1) performance in different self-control tasks should be correlated with one another, (2) these abilities should have a common neural substrate, and (3) a structural deficit in this substrate should adversely affect self-control.

Several lines of evidence (behavioral, neurocognitive, and clinical) suggest that different kinds of self-control share a common resource. Behavioral tests suggest a moderate correlation

Received June 4, 2010; revised Aug. 10, 2010; accepted Sept. 21, 2010.

This work was supported by National Institutes of Health Grants DA15179, DA022539, and DA024853 (E.D.L.), T32 MH17140 (G.T.), F30 DA021961 (K.B.), and MOI-RR-00865 (UCLA and G.C.R.C.); and endowments from the Katherine K. and Thomas P. Pike Chair in Addiction Studies and Marjorie M. Greene Trust.

Correspondence should be addressed to Edythe D. London, Semel Institute of Neuroscience and Human Behavior, UCLA, 760 Westwood Plaza, Los Angeles, CA 90024-1759. E-mail: elondon@mednet.ucla.edu.

G. Tabibnia's present address: Department of Social and Decision Sciences, Carnegie Mellon University, Pittsburgh, PA 15212.

J. R. Monterosso's present address: Department of Psychology, University of Southern California, Los Angeles, CA 90089.

A. R. Aron's present address: Department of Psychology, University of California, San Diego, CA 92093.

R. A. Poldrack's present address: Departments of Psychology and Neurobiology, University of Texas, Austin, TX 78712.

DOI:10.1523/JNEUROSCI.2859-10.2011

Copyright $\odot 2011$ the authors $\quad 0270-6474 / 11 / 314805-06 \$ 15.00 / 0$ between motor and cognitive inhibitory control (Miyake et al., 2000). However, behavioral evidence associating "cold" motor or cognitive inhibitory control with "hot" affective inhibitory control is limited. Affective and nonaffective inhibitory control measures are correlated in children (Carlson and Wang, 2007), but we are unaware of similar observations in healthy adults.

Neurocognitive studies have identified the ventrolateral prefrontal cortex, particularly the inferior frontal gyrus (IFG) in the right hemisphere, as playing an important role in both affective and nonaffective inhibitory control (Dillon and Pizzagalli, 2007; Lieberman, 2007). Within the right IFG, pars opercularis (IFGpo) is commonly associated with inhibitory control across tasks. Ability to inhibit a motor response is related to integrity of right IFGpo (Aron et al., 2004) and to its activation (Aron and Poldrack, 2006; Chikazoe et al., 2007; Leung and Cai, 2007). IFGpo is also activated during cognitive inhibition as in the colorword Stroop task (Leung et al., 2000) and during affective inhibitory control in affect regulation tasks (Ochsner et al., 2004; Kim and Hamann, 2007).

Deficits in inhibitory control and in IFG function are central aspects of many psychiatric disorders, such as depression (Johnstone et al., 2007), attention deficit hyperactivity disorder, obsessivecompulsive disorder, and addictions (Chambers et al., 2009), including methamphetamine dependence (Payer and London, 2009). Methamphetamine-dependent individuals exhibit poor motor inhibitory control (Monterosso et al., 2005) and emotion dysregulation (London et al., 2004). They also show structural (Thompson et al., 2004) and metabolic (London et al., 2004) abnormalities in many brain regions, including bilateral IFG. 
In short, most evidence relating motor and affective selfcontrol comes from neuroimaging studies reporting IFGpo activity in healthy individuals within a single self-control domain and from clinical studies demonstrating inhibitory control and IFG deficits in some disorders of self-control. Here, we examine whether affective and nonaffective inhibitory control abilities are related to one another and to IFGpo structure in the same healthy individuals, and whether a morphometric deficit in this common region accompanies behavioral deficits of inhibitory control.

\section{Materials and Methods}

Subjects. Forty-four healthy control adults (control: 21 females; average \pm SD age, $33.2 \pm 8.4$ years) and 43 abstinent methamphetaminedependent adults (METH: 19 females; average age, $33.4 \pm 9.0$ years) participated in a multistudy project. We acquired high-resolution magnetic resonance imaging (MRI) brain scans from all subjects. In subsets of subjects recruited for different studies, we also measured motor inhibitory control (25 control; $24 \mathrm{METH}$ ) and affect regulation ( 23 control; $22 \mathrm{METH}$ ).

Inclusion in the METH group required recent use of methamphetamine and no other illicit drugs (verified by urinalysis), $\geq 1$ year of using $\geq 1$ g of methamphetamine per week (self-report), and meeting Diagnostic and Statistical Manual of Mental Disorders, fourth edition (DSM-IV), criteria for amphetamine (methamphetamine) dependence by the Structured Clinical Interview for DSM-IV (First et al., 1995). Exclusionary criteria across groups were as follows: all current axis I diagnoses other than nicotine dependence; use of medications that affect the CNS; systemic disease; claustrophobia; pregnancy; HIV-seropositive status; hospitalization for psychiatric illness; and head trauma involving loss of consciousness and/or requiring hospitalization.

METH subjects resided at University of California, Los Angeles Hospital for 5-30 d. Abstinence from illicit drugs was confirmed by urine tests every other day and on each day of testing. The MRI scan, stopsignal task (SST), and emotion reappraisal task were administered on the 4th to 15th days of abstinence. Control subjects also underwent urine screens for drug use on each test day, but they participated on a nonresidential basis.

Withdrawal scores assessed in the METH sample using the Methamphetamine Withdrawal Questionnaire (MAWQ) (Zorick et al., 2010) were generally low at the time of the behavioral assessments. With a possible maximum score of 3.0 in each subscale, the mean scores closest to administration of the reappraisal task were as follows: 0.204 (range: $0-1.6$ ) for physical symptoms; 0.495 (range: $0-1.6$ ) for emotional symptoms; and 0.563 (range: $0-2.2$ ) for functional symptoms. For the SST, mean MAWQ scores were as follows: 0.096 (range: 0-0.5) for physical symptoms; 0.308 (range: $0-0.8$ ) for emotional symptoms; and 0.367 (range: $0-0.8$ ) for physical symptoms.

Tasks and measures. Motor inhibitory control was assessed using the SST (Logan, 1994), consisting of 256 trials (64 stop trials). Each trial began with a fixation dot appearing at the center of the screen for $500 \mathrm{~ms}$, followed by a target stimulus, "O" or "X," which remained for $2000 \mathrm{~ms}$. Subjects were to respond as quickly as possible with a left key-press for "O" or a right key-press for "X," but to try to stop themselves from pressing if the target was followed by a "stop-signal" tone ( $25 \%$ of trials). This signal was presented at a variable delay (the stop-signal delay) after the target stimulus appeared. After a successful Stop trial, the stop-signal delay was increased by $50 \mathrm{~ms}$, and after a failed Stop trial, it was decreased by $50 \mathrm{~ms}$, eventually titrating to a stop-signal delay resulting in $\sim 50 \%$ successful inhibition rate. All subjects reached a $42-58 \%$ successful inhibition rate. Stop-signal reaction time (SSRT), a measure of stopping ability, was calculated as the difference between the $n$th percentile Go reaction time (GoRT) and the average titrated stop-signal delay, where $n$ is the successful stopping rate; this method essentially corrects for deviations from $50 \%$ in the successful stopping rate (Logan, 1994). Because initial stop-signal delay values were often far from post-titration values, the computations were performed on the final 128 trials (32 Stop trials). Shorter SSRT indicates better inhibitory control.

Affective inhibitory control was assessed with the emotion reappraisal task (Ochsner et al., 2004). The experimental conditions of interest were
Look and Decrease. On Look trials, subjects were instructed to experience naturally (without trying to change) the emotional state elicited by the evocative pictures presented to them. On Decrease trials, they were instructed to decrease the intensity of their emotion by reinterpreting the picture in a non-negative way (e.g., a woman with facial bruises could be reinterpreted as an actress wearing makeup). The two conditions were matched for picture content (presence of face) and for subjective valence and arousal. To control for stimulus effects, each photograph was presented in the Look condition for half the subjects, and in the Decrease condition for the other half. The stimulus set included 32 highly arousing aversive pictures, selected based on normative ratings from the International Affective Picture System (Lang et al., 1993). On average, subjects' ratings of the negative pictures in the Look condition were positively correlated with the normative valence ratings of the pictures $(r=0.46$ in control; $r=0.44$ in METH), suggesting that both groups modulated their intensity ratings according to the intensity of the picture. Each trial began with a 2 s instruction screen ("LOOK" or "DECREASE"). A picture was then presented for $8 \mathrm{~s}$. Then subjects rated the strength of their negative emotion on a 7 -point Likert scale $(7=$ strongly negative). Finally, a blank screen was presented for $2 \mathrm{~s}$. Reappraisal success was calculated by the standard procedure, taking the difference in ratings between conditions (Look - Decrease), averaged across trials for each subject. Larger scores indicate better affect regulation.

Methamphetamine craving during 1 of the first $3 \mathrm{~d}$ of abstinence was measured in METH subjects using a self-report scale. Subjects were instructed to select, on a 10-point scale of 0 ("not at all") to 100 ("strongest ever"), the number that corresponded to their craving level for "the past 24 h."

Imaging. From each subject, we acquired a set of high-resolution T1weighted images (MPRAGE; repetition time $=1900 \mathrm{~ms}$; echo time $=$ $4.38 \mathrm{~ms} ; 15^{\circ}$ flip angle; $256 \times 256$ matrix; $1601 \mathrm{~mm}$ slices) on a $1.5 \mathrm{~T}$ Siemens Sonata scanner. The images were converted to Nifti format, their origins manually set at the anterior commissure, and preprocessed using statistical parametric mapping (SPM5; Wellcome Department of Imaging Neuroscience). Each brain was fitted to a gray matter probability map derived from $452 \mathrm{~T} 1$-weighted scans registered to the Montreal Neurological Institute space (www.loni.ucla.edu/ ICBM/ICBM_Probabilistic.html). To optimize intersubject registration, we used the DARTEL toolbox (modulated, $8 \mathrm{~mm}$ smoothing kernel) for within-group analyses (Ashburner, 2007) and the VBM5 toolbox (optimized, modulated, $10 \mathrm{~mm}$ smoothing kernel) for the between-groups analysis (Ashburner and Friston, 2005). The VBM5 homogeneity test was conducted, using SD across the sample, to identify and exclude outliers (in the sample of images preprocessed in VBM5, 3 control and $1 \mathrm{METH}$ were excluded; in the sample of images preprocessed in DARTEL, no subjects were excluded).

During the modulation step of preprocessing, voxel values in the segmented images were multiplied by the Jacobian determinants, which were obtained from the nonlinear deformation matrix created during spatial normalization. The purpose of modulation is to compensate for the warping effects in local volume by restoring the original volumes of segmented images (Keller et al., 2004). After modulation, the voxel values are a product of local brain volume and probability of a certain tissue type. While some researchers refer to this product as "gray matter volume," we use the term "gray matter intensity" to reflect the fact that the calculated values, which indicate volume, are generated from tissue probability maps in addition to Jacobian determinants. Modulated VBM provides a structurally and functionally meaningful measure of gray matter. Studies that have compared results from VBM analyses to those from visual and manual tracing have shown comparable results between the techniques (Whitwell, 2009). Functionally, deficits in these gray matter measures have been reported in numerous neurodegenerative (Whitwell, 2009) and psychiatric disorders, including panic disorder (Hayano et al., 2009 ) and attention deficit hyperactivity disorder (ADHD) (van't Ent et al., 2007).

The right inferior frontal gyrus pars opercularis region from the Harvard-Oxford Probabilistic Atlas $(p>0.25)$ was used as a mask for nonparametric analyses with FSL Randomize [www.fmrib.ox.ac.uk/fsl; 
A

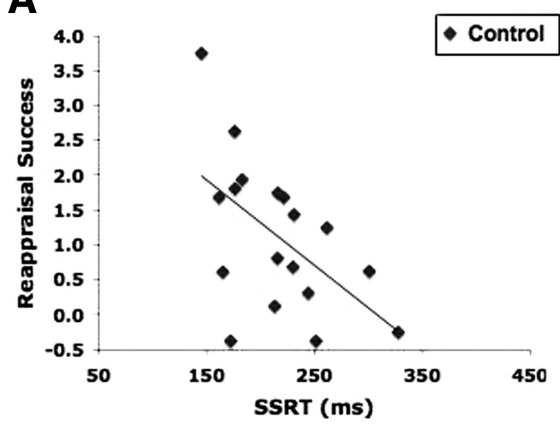

B

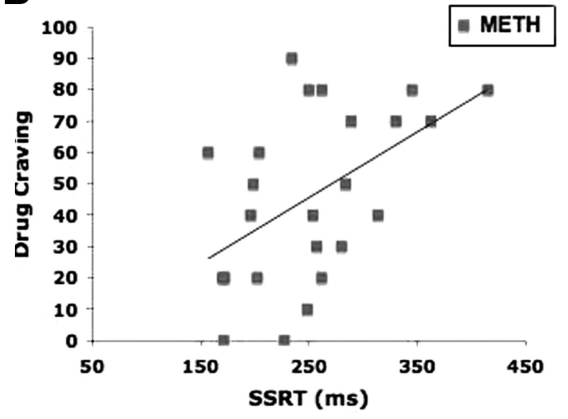

Figure 1. Affect regulation and drug craving were correlated with motor inhibitory control. $\boldsymbol{A}$, Control subjects with better motor inhibitory control, indexed by lower SSRT, exhibited greater reappraisal success (lower self-reported negative emotion, on a 7-point scale, with reappraisal than with passive looking). $\boldsymbol{B}$, METH subjects with better motor inhibitory control reported lower methamphetamine craving.
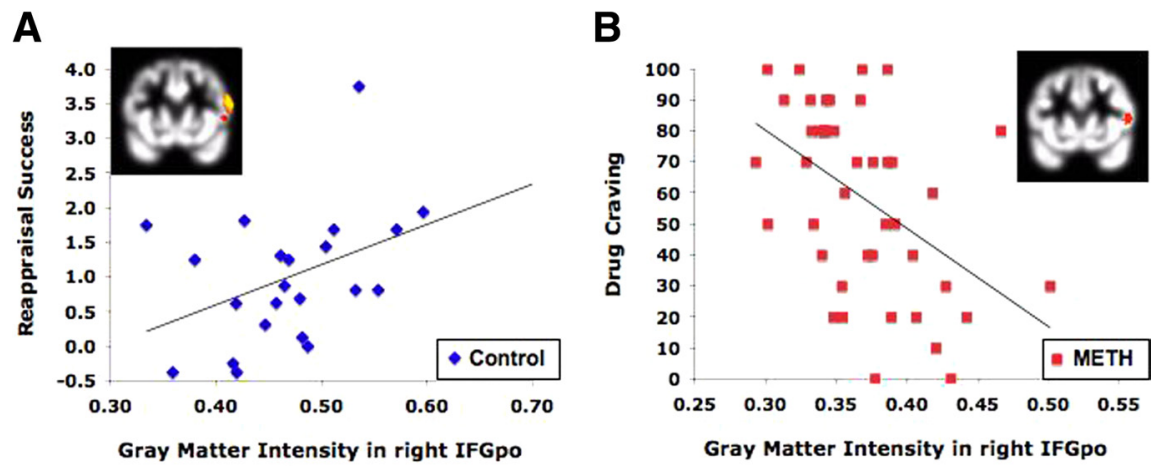

Figure 2. Affect regulation and drug craving were correlated with gray matter intensity in the right IFGpo. $\boldsymbol{A}$, Control subjects with higher gray matter intensity in right IFGpo exhibited greater reappraisal success $(r=0.50, p<0.05, n=23)$. $\boldsymbol{B}$, METH subjects with higher gray matter intensity in the same region reported lower craving for methamphetamine $(r=-0.48, p<$ $0.005, n=43$ ). Greater reappraisal success is indexed by lower self-reported negative emotion (7-point scale) with reappraisal than with passive looking. The $x$-axes represent Jacobian-transformed gray matter probabilities. Brain maps from standard permutation-based analyses illustrate foci where there was a significant correlation between behavior and gray matter intensity $\left(p_{(\mathrm{FWE})}<0.05\right)$. To prevent statistical bias, the gray matter values in the scatterplots were obtained from a leave-one-out cross-validation procedure.

5000 permutations, Threshold-Free Cluster Enhancement, corrected for Family Wise Error (FWE)]. To evaluate the specificity of results to the right IFGpo, we conducted post hoc analyses with additional masks from this atlas, namely the left IFGpo, the right IFG pars triangularis, and the right middle frontal gyrus (MFG), other lateral prefrontal regions that have been implicated in inhibitory control. In the post hoc analyses, we also used right and left pre-supplementary motor area (pre-SMA) regions of interest (ROIs) because of reports of an important role of this region in response inhibition (Aron et al., 2007a; Nachev et al., 2007) and in emotion reappraisal (Wager et al., 2008). We created this mask by using the SMA mask from the HarvardOxford atlas (which is also known as juxtapositional lobule cortex and includes pre-SMA) and excluding all voxels with $y<0$. We also conducted analyses with masks of right and left anterior cingulate cortex (ACC), SMA, subcallosal cortex, paracingulate gyrus, and ventromedial prefrontal cortex (VMPFC) - other medial prefrontal regions previously implicated in motor and affective inhibitory control (Wager et al., 2005, 2008; Urry et al., 2006).

Regression analyses on the behavioral measures were performed using SPSS16.0. The gray matter values for the scatterplots were obtained using a leave-one-out cross validation procedure (Ho et al., 2009): Using Randomize, a set of regression analyses were run with each subject left out, then the group mask (within the right IFGpo mask) determined from each of those analyses was used to extract the signal from the left-out individual, and those extracted values were then correlated with the behavioral measure.

\section{Results}

\section{Behavioral correlations}

Control subjects with better motor inhibitory control (lower SSRT) were better at affect regulation (greater reappraisal success) $(r=-0.55, p<0.05, n=18)$. This correlation in the small sample of METH subjects, although not statistically significant, was qualitatively similar to that observed among control subjects $(r=-0.46$, $p=0.15, n=11)$. METH subjects with better motor inhibitory control reported lower methamphetamine craving $(r=0.50$, $p<0.05, n=24)$. These results relate better motor inhibitory control to better affect regulation and lower craving (Fig. $1 A, B)$. In contrast, median GoRT was not correlated with reappraisal success in control subjects $(r=0.14)$ or with methamphetamine craving in METH subjects $(r=$ $-0.19)$. The affective processes studied are therefore related more specifically to motor inhibitory control than to the attentional and selection processes involved in Go trials.

Gray matter correlations with behavior Consistent with the critical role of right IFGpo in motor inhibitory control (Aron et al., 2004), control subjects $(n=25)$ with greater gray matter intensity in right IFGpo had better motor inhibitory control (lower SSRT) $\left(p_{(\mathrm{FWE})}=0.05\right)$ but showed no relation with GoRT ( $p_{(\mathrm{FWE})}>$ $0.5)$. Supporting the hypothesis that motor and affective inhibitory control share a neural substrate, greater gray matter intensity in right IFGpo was also related to better reappraisal success $\left(\mathrm{p}_{(\mathrm{FWE})}=0.05\right.$; $n=23)$ in control subjects and to lower methamphetamine craving $\left(\mathrm{p}_{(\mathrm{FWE})}=0.005 ; n=43\right)$ in METH subjects (Fig. $2 A, B$ ). In METH subjects, gray matter intensity in right IFGpo was not correlated with SSRT ( $\left.p_{(\mathrm{FWE})}>0.5, n=24\right)$, but it showed a trend toward a positive correlation with reappraisal success $\left(p_{(\mathrm{FWE})}<0.15, n=22\right)$.

Greater gray matter intensity in right MFG was not related to better motor inhibitory control $\left(p_{\mathrm{FWE})}>0.4\right)$ or greater reappraisal success $\left(p_{(\mathrm{FWE})}>0.6\right)$ in control subjects or to lower drug craving $\left(p_{(\mathrm{FWE})}>0.3\right)$ in METH subjects. In left IFGpo, gray matter intensity was not correlated with reappraisal success in control subjects $\left(p_{(\mathrm{FWE})}>0.2\right)$, but it was correlated with craving in METH subjects $\left(p_{(\mathrm{FWE})}<0.05\right)$, with greater gray matter intensity related to lower craving. In right pars triangularis, greater gray matter intensity was not related to better motor inhibitory control $\left(p_{(\mathrm{FWE})}>0.35\right)$ or better reappraisal success $\left(p_{(\mathrm{FWE})}>\right.$ $0.15)$ in control subjects, but it was related to lower drug craving $\left(p_{(\mathrm{FWE})}<0.05\right)$ in METH subjects.

In whole-brain analyses, even at a lenient threshold ( $p_{\text {(uncorrected) }}<$ 0.1 ), better motor inhibitory control in control subjects was not associated with greater gray matter intensity in any prefrontal region except right IFGpo [54 16 12] and left precentral gyrus $\left[\begin{array}{ll}-52 & 5\end{array} 2\right.$ 2]. Similarly, better reappraisal success in control sub- 
jects was not related to greater gray matter intensity in any prefrontal region except right IFGpo [54 16 12].

\section{METH versus control subjects}

In line with claims that methamphetamine-dependent individuals have neurocognitive deficits in self-control (Payer and London, 2009), gray matter intensity in right IFGpo was lower $\left(p_{(\mathrm{FWE})}<0.05\right)$ in METH $(n=42)$ than control $(n=41)$ subjects (Fig. 3), motor inhibitory control was worse $(p<0.05)$ in METH subjects $($ mean \pm SEM, $253.7 \pm 12.0 \mathrm{~ms} ; n=24)$ than control subjects (mean \pm SEM, $218.2 \pm 10.3 \mathrm{~ms} ; n=25)$ as previously reported (Monterosso et al., 2005), and reappraisal success was worse $(p<0.05)$ in METH subjects (mean \pm SEM, $0.42 \pm$ $0.21 ; n=22$ ) than control subjects (mean \pm SEM, 1.06 \pm 0.22 ; $n=23)$. GoRT showed no group difference $(p>0.5)$.

Gray matter intensity was greater in control than METH subjects in right MFG $\left(p_{(\mathrm{FWE})}<0.05\right)$ and right pars triangularis $\left(p_{(\mathrm{FWE})}<0.01\right)$ and marginally greater in left IFGpo $\left(p_{(\mathrm{FWE})}>\right.$ $0.07)$. Whole-brain analysis of group differences indicated greater gray matter intensity in control than METH subjects in right inferior frontal gyrus and in other parts of prefrontal cortex and temporal cortex (Morales et al., 2008).

\section{Post hoc analyses with medial prefrontal masks}

There was no significant correlation between gray matter intensity in right or left pre-SMA and either measure of self-control. Specifically, greater gray matter intensity in right or left pre-SMA did not relate to better motor inhibitory control $\left(p_{(\mathrm{FWE})}>0.7\right.$ for right; $p_{(\mathrm{FWE})}>0.5$ for left) or greater reappraisal success $\left(p_{(\mathrm{FWE})}>0.7\right.$ for right; $p_{\text {(FWE })}>0.7$ for left). Better motor inhibitory control did not relate to greater gray matter intensity in any of the other medial prefrontal ROIs ( $p_{\text {(FWE) }}>0.3$ for all analyses), except in left subcallosal gyrus, where it showed a trend toward a significant correlation $\left(p_{(\mathrm{FWE})}>\right.$ 0.12 ). Reappraisal success did not relate to greater gray matter intensity in any of these remaining medial prefrontal ROIs, namely right and left ACC, right and left SMA, right and left subcallosal cortex, right and left paracingulate gyrus, or right and left $\operatorname{VMPFC}\left(p_{(\mathrm{FWE})}>0.4\right.$ for all analyses $)$.

\section{Discussion}

In healthy subjects, success in motor inhibitory control and in affect regulation were positively correlated, and success on both tasks was positively correlated with gray matter intensity in overlapping areas of right IFGpo. Furthermore, motor inhibitory control and gray matter intensity in right IFGpo correlated with methamphetamine craving in METH subjects, who exhibited deficits in inhibitory control and in gray matter integrity in this region.

These results support previous reports suggesting a role of the right IFG in inhibitory control. Successfully withholding a prepotent motor response depends on structural integrity of right IFGpo and activates this region (Aron et al., 2004; Leung and Cai, 2007; Forstmann et al., 2008). Conjunction and meta-analyses have identified activation in right IFGpo during both motor (e.g., SST, go/no-go) and cognitive (e.g., Wisconsin Card Sorting Task, flanker task) inhibitory control, with some overlap between the two (Bunge et al., 2002; Buchsbaum et al., 2005; Nee et al., 2007).
Importantly, downregulation of negative emotions has also been associated with increased activity in right IFG (Lieberman et al., 2007), including right IFGpo (Hariri et al., 2000; Ochsner et al., 2004; Phan et al., 2005). Moreover, consistent with the purported role of IFG in inhibitory control across domains, activating right IFG during motor inhibitory control has an unintended inhibitory effect on emotion-related amygdala activation (Berkman et al., 2009). Our findings complement these previous reports and suggest that gray matter in an overlapping region of right IFG may support both affective and nonaffective inhibitory control.

A recent study suggests that right IFGpo may participate in control of craving (Volkow et al., 2010). Although we did not measure craving control explicitly, the correlation of gray matter intensity in right IFGpo with self-reported spontaneous craving is consistent with a role of this region in downregulation of craving. Greater gray matter intensity in this region was associated with lower methamphetamine craving in hospitalized subjects to whom the drug was unavailable and who would optimally downregulate craving to cope. However, the additional correlation of craving with left IFGpo and right pars triangularis suggests an association of craving with overall gray matter degradation or with addiction severity in general. The absence of correlation between craving and right MFG, however, weakens that possibility.

Impaired motor inhibitory control has been related to deficits in complex and affective self-control across several psychiatric disorders. Boys with ADHD have slower SSRT along with emotion dysregulation (Nigg, 2001). Cocaine abusers have slower SSRT (Fillmore and Rush, 2002), and neural activity during stopping in this group correlates with self-reported ability to regulate emotions ( $\mathrm{Li}$ et al., 2008). Poor stopping has also been associated with some eating and anxiety disorders (Nederkoorn et al., 2004; Chamberlain and Sahakian, 2007). Investigators of the neurocognitive underpinnings of these disorders often use simple motor inhibitory control tasks, with the assumption that these tasks share psychological and neural components with the more complex processes of impulsivity and affect regulation that are compromised in the disorder. Our results support this assumption and the notion that a deficit in motor inhibitory control may be a risk factor for addiction and other psychiatric disorders of self-control.

Despite differences between motor inhibitory control and affect regulation, it is plausible that they share a common substrate. The IFG directly projects to the subthalamic nucleus, which can inhibit motor and limbic responses via the basal ganglia (see Aron 
et al., 2007b). Alternatively, the IFG may support a broader attention-orienting or information-selection function in motor (Chambers et al., 2009) and affective (Wager et al., 2008) selfcontrol. Whatever the specific computation of the right IFG, our results suggest that it supports motor inhibitory control in the stop-signal task and affect inhibitory control in the reappraisal task.

Undoubtedly, the right IFG is not the only region important for inhibitory control, as other regions within the prefrontal cortex have also been implicated (Cohen and Lieberman, 2010). However, right IFGpo seems to be a particularly likely region to support inhibitory control across psychological domains. Among all ROIs used in this study, right IFGpo was the only one in which gray matter intensity correlated with all three behavioral measures (motor and affective inhibitory control, and craving). In fact, SSRT and reappraisal success were not correlated with gray matter intensity in any other ROI. These results do not conflict with reports of neural activation in other prefrontal regions during inhibitory control, as greater gray matter intensity in a region is not necessarily coupled with greater or lower activation in that region (Jacobson et al., 2010). More notably, our results are consistent with lesion studies showing that the right IFGpo is one of the only regions critical for motor inhibitory control (Aron et al., 2004).

Although some have suggested an exclusively attention-orienting role of right IFG in motor inhibitory control (Hampshire et al., 2010), there are several lines of recent evidence arguing for the role of right IFG as not merely one of monitoring or orienting attention, but as implementing inhibitory control itself. A recent study used TMS to compare disruption of the right IFG (pars opercularis region) with disruption of the more dorsal right inferior frontal juncture (IFJ) region (Verbruggen et al., 2010). Disruption of both regions affected response inhibition speed, but apparently by affecting different processes. It was argued that the right IFJ implements attentional orienting, while the more ventral sector of right IFG (pars opercularis region) implements inhibitory control. This is highly consistent with a recent functional MRI study, which found that IFJ was activated in relation to the "oddball effect" of infrequent NoGo stimuli, while the more ventral IFG region was activated by the putative response inhibition requirement when controlling for the oddball effect (Chikazoe et al., 2009).

Strong evidence for an inhibitory control function for IFG was recently provided by a paired-pulse TMS (transcranial magnetic stimulation) study, which showed that when action countermanding was required, the connection between right IFG and M1 became strongly inhibitory (Buch et al., 2010). Further, a recent study with the stop signal paradigm, which recorded from the right IFG in patients being evaluated for epilepsy, reported a significantly greater response for successful than unsuccessful stop trials (Swann et al., 2009). This comparison controls for the oddball effect. Importantly, in that study the increased neural response on successful stop trials was within the timescale of SSRT, and it occurred in the beta-frequency band. Given that increases in the beta-frequency band are strongly associated with stopping movement, including within the primary motor cortex (Swann et al., 2009) and the subthalamic nucleus (Kühn et al., 2004) (which may participate in a structurally connected functional circuit with the right IFG and dorsomedial frontal cortex) (Aron and Poldrack, 2006; Aron et al., 2007a), these findings are much better reconcilable with an inhibitory control function for right IFG rather than merely a monitoring/attentional-orienting role.

All current axis I diagnoses, except amphetamine (methamphetamine) dependence and nicotine dependence, were exclu- sionary for both groups, but a greater proportion of METH than control participants were smokers. It is therefore plausible that smoking status may have affected the behavioral and gray matter assessments, especially in the METH group. Our results and the literature, however, do not support this possibility. As in a prior report (Monterosso et al., 2005), SSRT did not differ between smoking $(n=8)$ and nonsmoking $(n=17)$ control participants $(p=0.71)$ in the current study. We obtained similar results $(p=$ 0.32 ) in a larger sample of control participants (26 smokers, 25 nonsmokers) who completed the SST for another study in our laboratory. In this larger sample, comparing smoker METH participants $(n=31)$ to smoker control participants $(n=26)$ still yielded a reliable group difference in SSRT $(p=0.005)$. The effect of smoking on reappraisal success is more difficult to assess, due to the small sample size. Reappraisal success did not differ between smoking $(n=7)$ and nonsmoking $(n=16)$ control participants $(p=0.88)$. Comparing smoker METH participants $(n=22)$ to smoker control participants $(n=7)$ yielded a trend toward a significant difference in reappraisal success $(p=0.14)$, suggesting that this question may warrant further study.

The results presented here demonstrate that "cold" motor and "hot" affective self-control involve a common substrate in the IFG, which is compromised in stimulant dependence, a disorder that features self-control deficits and craving. Our findings suggest that a shared neurocognitive system subserves different kinds of self-control, although the specific computation performed by this system is still unclear. An important implication of these findings is that defects in an IFG self-control system may underlie different disorders of self-control (Chamberlain and Sahakian, 2007). Conversely, strengthening this system in one psychological domain may enhance self-control in another domain (Muraven and Baumeister, 2000). Further research is needed to support these hypotheses.

\section{References}

Aron AR, Poldrack RA (2006) Cortical and subcortical contributions to stop signal response inhibition: role of the subthalamic nucleus. J Neurosci 26:2424-2433

Aron AR, Robbins TW, Poldrack RA (2004) Inhibition and the right inferior frontal cortex. Trends Cogn Sci 8:170-177.

Aron AR, Behrens TE, Smith S, Frank MJ, Poldrack RA (2007a) Triangulating a cognitive control network using diffusion-weighted magnetic resonance imaging (MRI) and functional MRI. J Neurosci 27:3743-3752.

Aron AR, Durston S, Eagle DM, Logan GD, Stinear CM, Stuphorn V (2007b) Converging evidence for a fronto-basal-ganglia network for inhibitory control of action and cognition. J Neurosci 27:11860-11864.

Ashburner J (2007) A fast diffeomorphic image registration algorithm. Neuroimage 38:95-113.

Ashburner J, Friston KJ (2005) Unified segmentation. Neuroimage 26:839-851.

Berkman ET, Burklund L, Lieberman MD (2009) Inhibitory spillover: intentional motor inhibition produces incidental limbic inhibition via right inferior frontal cortex. Neuroimage 47:705-712.

Buch ER, Mars RB, Boorman ED, Rushworth MF (2010) A network centered on ventral premotor cortex exerts both facilitatory and inhibitory control over primary motor cortex during action reprogramming. J Neurosci 30:1395-1401

Buchsbaum BR, Greer S, Chang WL, Berman KF (2005) Meta-analysis of neuroimaging studies of the Wisconsin card-sorting task and component processes. Hum Brain Mapp 25:35-45.

Bunge SA, Dudukovic NM, Thomason ME, Vaidya CJ, Gabrieli JD (2002) Immature frontal lobe contributions to cognitive control in children: evidence from fMRI. Neuron 33:301-311.

Carlson SM, Wang TS (2007) Inhibitory control and emotion regulation in preschool children. Cogn Dev 22:489-510.

Chamberlain SR, Sahakian BJ (2007) The neuropsychiatry of impulsivity. Curr Opin Psychiatry 20:255-261. 
Chambers CD, Garavan H, Bellgrove MA (2009) Insights into the neural basis of response inhibition from cognitive and clinical neuroscience. Neurosci Biobehav Rev 33:631-646.

Chikazoe J, Konishi S, Asari T, Jimura K, Miyashita Y (2007) Activation of right inferior frontal gyrus during response inhibition across response modalities. J Cogn Neurosci 19:69-80.

Chikazoe J, Jimura K, Asari T, Yamashita K, Morimoto H, Hirose S, Miyashita Y, Konishi S (2009) Functional dissociation in right inferior frontal cortex during performance of go/no-go task. Cereb Cortex 19:146-152.

Cohen JR, Lieberman, MD (2010) The common neural basis of exerting self-control in multiple domains. In: Self-control (Trope Y, Hassin R, Ochsner KN, eds), pp 141-160. New York: Oxford UP.

Dillon DG, Pizzagalli DA (2007) Inhibition of action, thought, and emotion: a selective neurobiological review. Appl Prev Psychol 12:99-114.

Fillmore MT, Rush CR (2002) Impaired inhibitory control of behavior in chronic cocaine users. Drug Alcohol Depend 66:265-273.

First MB, Spitzer RL, Gibbon M, Williams JBW (1995) The structured clinical interview for DSM-IV axis I disorders (SCID-IP). Washington, DC: American Psychiatric Press.

Forstmann BU, Jahfari S, Scholte HS, Wolfensteller U, van den Wildenberg WP, Ridderinkhof KR (2008) Function and structure of the right inferior frontal cortex predict individual differences in response inhibition: a model-based approach. J Neurosci 28:9790-9796.

Hampshire A, Chamberlain SR, Monti MM, Duncan J, Owen AM (2010) The role of the right inferior frontal gyrus: inhibition and attentional control. Neuroimage 50:1313-1319.

Hariri AR, Bookheimer SY, Mazziotta JC (2000) Modulating emotional responses: effects of a neocortical network on the limbic system. Neuroreport 11:43-48.

Hayano F, Nakamura M, Asami T, Uehara K, Yoshida T, Roppongi T, Otsuka T, Inoue T, Hirayasu Y (2009) Smaller amygdala is associated with anxiety in patients with panic disorder. Psychiatry Clin Neurosci 63:266-276.

Ho TC, Brown S, Serences JT (2009) Domain general mechanisms of perceptual decision making in human cortex. J Neurosci 29:8675-8687.

Jacobson S, Kelleher I, Harley M, Murtagh A, Clarke M, Blanchard M, Connolly C, O'Hanlon E, Garavan H, Cannon M (2010) Structural and functional brain correlates of subclinical psychotic symptoms in 11-13 year old schoolchildren. Neuroimage 49:1875-1885.

Johnstone T, van Reekum CM, Urry HL, Kalin NH, Davidson RJ (2007) Failure to regulate: counterproductive recruitment of top-down prefrontalsubcortical circuitry in major depression. J Neurosci 27:8877-8884.

Keller SS, Wilke M, Wieshmann UC, Sluming VA, Roberts N (2004) Comparison of standard and optimized voxel-based morphometry for analysis of brain changes associated with temporal lobe epilepsy. Neuroimage 23:860-868.

Kim SH, Hamann S (2007) Neural correlates of positive and negative emotion regulation. J Cogn Neurosci 19:776-798.

Kühn AA, Williams D, Kupsch A, Limousin P, Hariz M, Schneider GH, Yarrow K, Brown P (2004) Event-related beta desynchronization in human subthalamic nucleus correlates with motor performance. Brain 127:735-746.

Lang PJ, Greenwald MK, Bradley MM, Hamm AO (1993) Looking at pictures: affective, facial, visceral, and behavioral reactions. Psychophysiology 30:261-273.

Leung HC, Cai W (2007) Common and differential ventrolateral prefrontal activity during inhibition of hand and eye movements. J Neurosci 27:9893-9900.

Leung HC, Skudlarski P, Gatenby JC, Peterson BS, Gore JC (2000) An event-related functional MRI study of the stroop color word interference task. Cereb Cortex 10:552-560.

Li CS, Huang C, Yan P, Bhagwagar Z, Milivojevic V, Sinha R (2008) Neural correlates of impulse control during stop signal inhibition in cocainedependent men. Neuropsychopharmacology 33:1798-1806.

Lieberman MD (2007) Social cognitive neuroscience: a review of core processes. Annu Rev Psychol 58:259-289.

Lieberman MD, Eisenberger NI, Crockett MJ, Tom SM, Pfeifer JH, Way BM (2007) Putting feelings into words: affect labeling disrupts amygdala activity to affective stimuli. Psychol Sci 18:421-428.

Logan, G (1994) On the ability to inhibit thought and action. In: Inhibitory processes in attention, memory and language (Dagenbach D, Carr TH, eds), pp 189-239. New York: Academic.

London ED, Simon SL, Berman SM, Mandelkern MA, Lichtman AM, Bramen
J, Shinn AK, Miotto K, Learn J, Dong Y, Matochik JA, Kurian V, Newton T, Woods R, Rawson R, Ling W (2004) Mood disturbances and regional cerebral metabolic abnormalities in recently abstinent methamphetamine abusers. Arch Gen Psychiatry 61:73-84.

Miyake A, Friedman NP, Emerson MJ, Witzki AH, Howerter A, Wager TD (2000) The unity and diversity of executive functions and their contributions to complex "frontal lobe" tasks: a latent variable analysis. Cogn Psychol 41:49-100.

Monterosso JR, Aron AR, Cordova X, Xu J, London ED (2005) Deficits in response inhibition associated with chronic methamphetamine abuse. Drug Alcohol Depend 79:273-277.

Morales AM, Berman S, Chakrapani S, Lee B, Zorick T, Tabibnia G, London ED (2008) A voxel-based morphometry study of gray matter volume deficits in methamphetamine abusing subjects. Soc Neurosci Abstr 34:455.4.

Muraven M, Baumeister RF (2000) Self-regulation and depletion of limited resources: does self-control resemble a muscle? Psychol Bull 126:247-259.

Nachev P, Wydell H, O'Neill K, Husain M, Kennard C (2007) The role of the pre-supplementary motor area in the control of action. Neuroimage 36 [Suppl 2]:T155-T163.

Nederkoorn C, Van Eijs Y, Jansen A (2004) Restrained eaters act on impulse. Pers Individ Dif 37:1651-1658.

Nee DE, Wager TD, Jonides J (2007) Interference resolution: insights from a meta-analysis of neuroimaging tasks. Cogn Affect Behav Neurosci 7:1-17.

Nigg JT (2001) Is ADHD a disinhibitory disorder? Psychol Bull 127: 571-598.

Ochsner KN, Ray RD, Cooper JC, Robertson ER, Chopra S, Gabrieli JD, Gross JJ (2004) For better or for worse: neural systems supporting the cognitive down- and up-regulation of negative emotion. Neuroimage 23:483-499.

Payer D, London ED (2009) Methamphetamine and the brain: findings from brain imaging studies. In: Methamphetamine: a guide for practitioners (Roll J, Rawson R, Ling W, Shoptaw S, eds), pp 61-91. New York: Guilford.

Phan KL, Fitzgerald DA, Nathan PJ, Moore GJ, Uhde TW, Tancer ME (2005) Neural substrates for voluntary suppression of negative affect: a functional magnetic resonance imaging study. Biol Psychiatry 57:210-219.

Swann N, Tandon N, Canolty R, Ellmore TM, McEvoy LK, Dreyer S, DiSano M, Aron AR (2009) Intracranial EEG reveals a time- and frequencyspecific role for the right inferior frontal gyrus and primary motor cortex in stopping initiated responses. J Neurosci 29:12675-12685.

Thompson PM, Hayashi KM, Simon SL, Geaga JA, Hong MS, Sui Y, Lee JY, Toga AW, Ling W, London ED (2004) Structural abnormalities in the brains of human subjects who use methamphetamine. J Neurosci 24:6028-6036.

Urry HL, van Reekum CM, Johnstone T, Kalin NH, Thurow ME, Schaefer HS, Jackson CA, Frye CJ, Greischar LL, Alexander AL, Davidson RJ (2006) Amygdala and ventromedial prefrontal cortex are inversely coupled during regulation of negative affect and predict the diurnal pattern of cortisol secretion among older adults. J Neurosci 26:4415-4425.

van 't Ent D, Lehn H, Derks EM, Hudziak JJ, Van Strien NM, Veltman DJ, De Geus EJ, Todd RD, Boomsma DI (2007) A structural MRI study in monozygotic twins concordant or discordant for attention/hyperactivity problems: evidence for genetic and environmental heterogeneity in the developing brain. Neuroimage 35:1004-1020.

Verbruggen F, Aron AR, Stevens MA, Chambers CD (2010) Theta burst stimulation dissociates attention and action updating in human inferior frontal cortex. Proc Natl Acad Sci U S A 107:13966-13971.

Volkow ND, Fowler JS, Wang GJ, Telang F, Logan J, Jayne M, Ma Y, Pradhan K, Wong C, Swanson JM (2010) Cognitive control of drug craving inhibits brain reward regions in cocaine abusers. Neuroimage 49:25362543 .

Wager TD, Sylvester CY, Lacey SC, Nee DE, Franklin M, Jonides J (2005) Common and unique components of response inhibition revealed by fMRI. Neuroimage 27:323-340.

Wager TD, Davidson ML, Hughes BL, Lindquist MA, Ochsner KN (2008) Prefrontal-subcortical pathways mediating successful emotion regulation. Neuron 59:1037-1050.

Whitwell JL (2009) Voxel-based morphometry: an automated technique for assessing structural changes in the brain. J Neurosci 29:9661-9664.

Zorick T, Nestor L, Miotto K, Sugar C, Hellemann G, Scanlon G, Rawson R, London ED (2010) Withdrawal symptoms in abstinent methamphetamine-dependent subjects. Addiction 105:1809-1818 